\begin{tabular}{c} 
International Journal of Engineering \& Technology, $7(3)(2018)$ 1609-1614 \\
International Journal of Engineering \& Technology \\
SPC \\
Website: $\begin{array}{c}\text { ww. sciencepubco.com/index.php/IJET } \\
\text { doi: } 10.14419 / \text { ijet.v7i3.15041 } \\
\text { Research paper }\end{array}$ \\
\hline
\end{tabular}

\title{
Biofeedback of states of anxiety through automated detection processes using different technologies
}

\author{
Benjamin Maraza Quispe ${ }^{1 *}$, Luis Alfaro Casas ${ }^{2}$, Olga Melina Alejandro Oviedo ${ }^{3}$, Crisia Vivanco Chavez ${ }^{4}$, \\ Yony Jiménez Villegas ${ }^{5}$, Simón Choquehuayta Palomino ${ }^{6}$, José Herrera Quispe ${ }^{7}$, Nicolas Caytuiro Silva ${ }^{8}$ \\ ${ }^{1}$ Department of Computer Science, San Agustín University, Arequipa, Perú \\ ${ }^{2}$ Department of Computer Science, San Agustín University, Arequipa, Perú \\ ${ }^{3}$ Academic Department of Education Sciences, San Agustín University, Arequipa, Perú \\ ${ }^{4}$ Department of Computer Science, San Agustín University, Arequipa, Perú \\ ${ }^{5}$ Academic Department of Education Sciences, Pedro Ruiz Gallo University, Lambayeque, Perú \\ ${ }^{6}$ Department of Computer Science, San Agustín University, Arequipa, Perú \\ ${ }^{7}$ Department of Computer Science, San Agustín University, Arequipa, Perú \\ ${ }^{8}$ High Performance College of Arequipa, Arequipa, Perú \\ *Corresponding author E-mail:benjimaraza@gmail.com
}

\begin{abstract}
In this work, a system model is proposed, which applies biofeedback techniques, through automatic detection procedures, using different technologies, constituted by a system formed by a prototype based on a set of "Arduino" microcontrollers, which monitor the levels of anxiety, using as a measurement technique, the Body Temperature Variables, and the Galvanic Skin Response. The measurement is validated by comparing Zung's self-report and depressive symptoms scale, which offers diagnostic approaches, which comprise most of the characteristics of anxiety or depression, involving affective, physiological and psychological aspects, with a range of punctuations. From 20 to 80 points. The presence of anxiety or depression is assumed with scores higher than $50 \%$; controlled in an environment of the Android operating system, which interprets the data obtained, analyzing and sending them from an "Arduino Uno" plate, to display them through an application (App), using the App Inventor platform, which receives the signals from anxiety levels, using radiofrequency waves with Bluetooth technology, in order to provide self-management treatment of timely and effective anxiety levels to achieve the improvement of quality of life. This research work is motivated, because the anxiety sustained for long periods of time, can be a risk factor for diseases, lack of productivity and work absences, therefore, it can be considered as a factor that causes significant economic losses. The results obtained were satisfactory, since it was possible to considerably reduce the levels of anxiety, through the application of the techniques of the model that applies biofeedback techniques.
\end{abstract}

Keywords: Anxiety; Anxiety and Depression Test; Biofeedback; Microcontrollers; Neural Networks.

\section{Introduction}

The evolution of technology in recent years, has grown exponentially since the advent of desktop computers, but this evolution has been even faster with the appearance of the first "mobile devices", a few years ago, times in which, nobody believed that you could have computers in the palm of your hand, allowing users to surf the internet, access social networks, send and receive files, to communicate globally worldwide [2]. Technology and the internet, has generated that every time we are more interconnected among us, arising the need to have everything controlled with our mobile device.

Neural Networks are a computational branch of Artificial Intelligence based on the neural model of human beings. The results of its application today have penetrated homes through appliances that carry this technology. Likewise, smart mobile devices are a growing technology and part of the current research in engineering focuses on how to implement cybernetics, software and telecommunications in the terminals mentioned [3].
Arduino is a free hardware platform, based on an electronic board that has a Micro controller and an integrated development environment, designed to be used in multidisciplinary projects. Its programming demands basic programming concepts in the $\mathrm{C} / \mathrm{C}++$ languages [1].

App Inventor, is a software development environment created by Google Labs, for the development of applications for the Android operating system. The user can, visually and from a set of basic tools, link a series of blocks to create the application. The system is free and can be easily downloaded from the web. The applications created with App Inventor are limited by their simplicity, although they allow covering a large number of basic needs in a mobile device.

This research aims to propose, design and implement an appropriate architecture model through an embedded system with Arduino microcontrollers, for the proper functioning of the system and at the same time identify the elements that make it up, designing a friendly and interactive interface of easy manipulation for the user, enabling the recognition of anxiety levels, based on the analysis of the skin's galvanic response, body temperature and cardiac rhythms. To provide biofeedback techniques through applications designed and implemented for this purpose. 


\section{Research fundament}

\subsection{Biofeedback}

Biofeedback refers to a group of therapeutic procedures that use electronic instruments for accurate measurement, which offer people information about their neuromuscular and autonomic activity, in the form of reinforcement signals of different types, in order to achieve voluntary control over their physiological processes, controlling first the external signals and then the internal physiological inputs [10].

In other words, it is the process by which the physiological information, usually imperceptible, becomes observable through the use of instrumentation with the purpose of altering a particular response.

\subsection{Most used instruments for applying biofeedback}

There are four main types of biofeedback instrumentation available for clinical use [11].

Temperature measurement: It detects the external temperature of the body, usually of the hands, or feet. The instrumentation that does this is often called a thermal unit or thermal examiner. The external temperature of the periphery can give an indication of anxiety or relaxation.

Galvanic Skin Response: It examines the amount of conduction or resistance to the passage of a small electric current through the skin. For experimental and research purposes, psychologists have been measuring electro-dermal activity since the beginning of the century, [8]. In 1967, Gary Schwartz showed that one can learn to control galvanic skin responses (GSR).

Electromyography: It detects the contraction or tension of the muscles. When the muscles are not contracted, it therefore provides a measure of relaxation. This instrumentation is called electro myograph or EMG, it is often the favourite instrument for relaxation assisted by biofeedback

Electroencephalography: it detects the type and amount of brain waves that are occurring. This instrument is commonly referred to as an electroencephalograph or EEG. It has been used in medicine for diagnostic purposes since 1920.

\subsection{Uses of biological feedback in psychotherapy}

There are different uses of Biofeedback in Psychotherapy: [11] Primary treatment with auxiliary psychotherapy: Anxiety and psychosomatic disorders. Areas with problems that may emerge low self-esteem need for cognitive restructuring, need for help to restructure the lifestyle as the symptoms diminish.

Relaxation treatment together with psychotherapy: Precursor of therapies that use free association, or visual images. Phobias and Anxiety, psychosomatic disorders and those related to anxiety.

As a control for psychotherapy: Diagnosis and establishment of problem areas in the initial interview. When the signal appears that anxiety is reaching a high level of resistance.

Personal growth: self-regulation applied for preventive and development goals. Possible Goals: Feeling relaxed and comfortable. Energy changes. Creativity increase Become more alert to initial voltage signals. Recognition and minimization of anxiety in daily life.

\subsection{Artificial neuronal networks}

Neural networks are a very simple implementation of a local behavior observed in our brains [5]. The brain is composed of neurons, which are individual elements of processing [6]. Nowadays, neural networks are trained to solve problems that are difficult for conventional computers or humans [7]. Each neuron is connected to another neuron by means of an adjustment weight represented by the letter w; the first sub-index indicates the destination neuron, while the second sub-index indicates the origin neuron as can be seen in the following image [9]

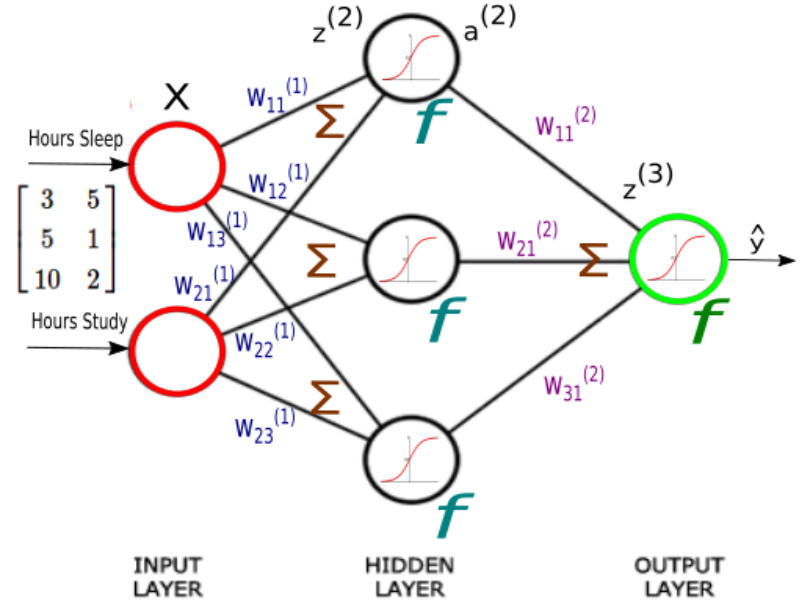

Fig. 1: Components of an Artificial Neural Network [9].

\section{Methodology and experimental procedure}

\subsection{Justification}

Anxiety, sustained for long periods of time, generates diseases, lack of performance and absences from work, therefore it is considered as a generator of very important economic losses. On the other hand due to the high indexes of anxiety that were detected in a preliminary investigation developed in a first stage of the investigation with a group of 50 students of the High Performance College of Arequipa, in which they were detected through the application of the anxiety and depression test standardized by Zung, [4]. These high levels of anxiety were confirmed through the measurement developed with our prototype, using the application of different techniques, such as Galvanic Skin Response and body temperature, for this reason it is necessary to receive timely and effective treatment with the purpose of being able to improve the standards of quality of life of the people.

\subsection{Hypothesis}

"The application of biofeedback techniques implemented through the automation of processes and use of available technologies at our reach, contribute to reduce high levels of anxiety levels, providing self-control techniques and timely self-regulation reaching relaxation states in order to achieve a better quality of life"

\subsection{Research question}

To what extent is it possible to reduce high anxiety rates through the application of biofeedback techniques implemented through the automation of processes and the use of available technologies?

\subsection{General purpose}

Propose a model that uses techniques for biofeedback of anxiety states, through automatic detection procedures using different available technologies, providing timely and effective self-control treatments to achieve a better quality of life.

The following figure shows the prototype culminated according to the proposed research objective: 


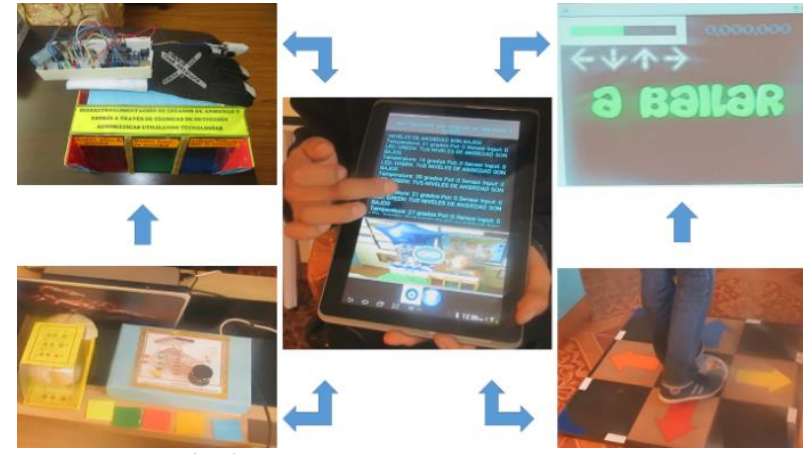

Fig. 2: Prototype Completed in Operation.

Source: self-made

\subsection{Specific objectives}

Design and implement an application (App), whose interface will serve as an intermediary between the results of the measurement and the self-control treatment to reduce high levels of anxiety.

Design and implement a module called "Dance Anti-Anxiety Pad Revolution" in order to apply anti-anxiety dynamics for bodily events.

Validate the results of the methodology implemented through the experimentation and study of the state of the art.

\subsection{Expected results}

Through the present investigation, it is expected to considerably reduce the anxiety levels of the population of students considered in the sample.

\section{Process}

The research was developed with an experimental group of $50 \mathrm{stu}-$ dents who were applied the standardized anxiety test by Zung and our prototype, and in the two measurements it was possible to determine high levels of anxiety, so that this same sample of students was proceeded to apply Biofeedback techniques, through the automation of processes and use of available technologies: A module "Dance Anti-Anxiety Pad Revolution", implemented for the application of anti-anxiety body dynamics, and an App implemented for that purpose; Below the procedures developed are detailed:

\subsection{Biofeedback through the application (APP)}

The first figure shows the main interface of the mobile application designed for interaction and surfing through the App, contains the biofeedback sections of anxiety states, and the second section that allows directing to the monitoring interface of anxiety levels.

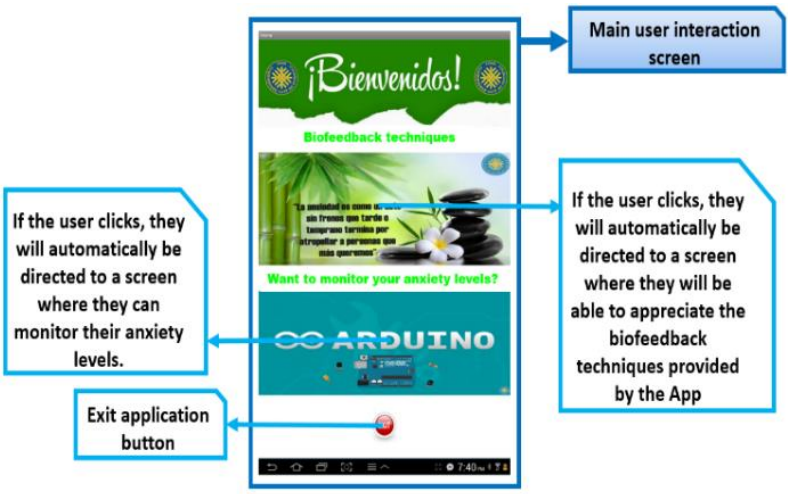

Fig. 3: Main Interface of the Application (App).
The second figure shows the interface of real-time monitoring of anxiety levels, which are sent from the prototype to the App by means of radiofrequency waves, in addition you can also see the main buttons

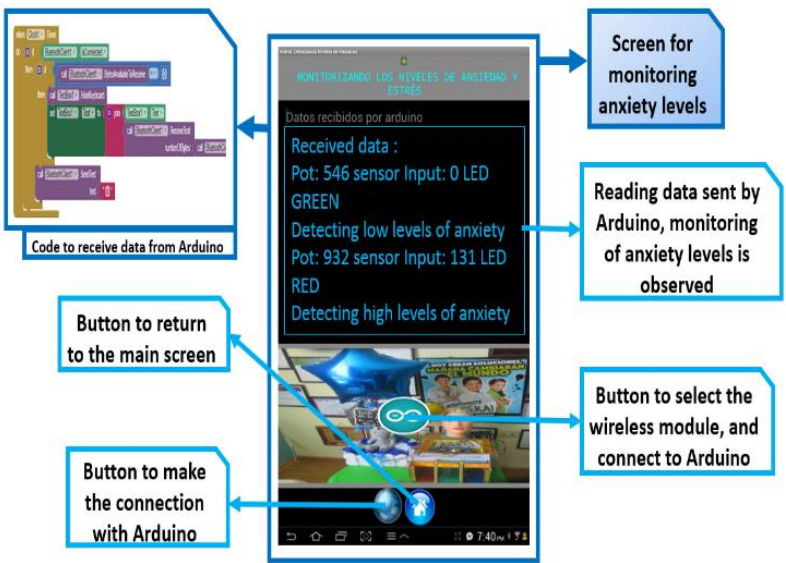

Fig. 4: Monitoring of Data Sent from the Prototype Through an App.

Source: self-made.

The following graphical interface shows the Biofeedback techniques that were designed and implemented and the way they are applied. However, our goal is not to only make an App, but to know the impact of this by offering an alternative for biofeedback of anxiety levels.

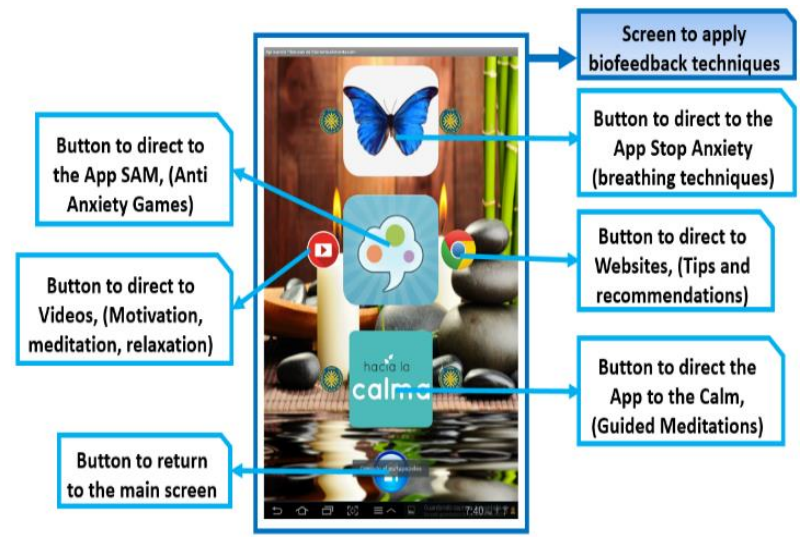

Fig. 5: Biofeedback Techniques through Mobile Applications.

Source: self-made

\subsection{Biofeedback through the module dance anti-anxiety pad revolution}

The proposal called "Dance Anti-Anxiety Pad Revolution" is according to the characteristics of the users, whose purpose is to apply anti-anxiety dynamics for bodily events, which consist of anti-anxiety bioenergetics therapies.

In Figure 6, the circuit diagram of the Dance Anti-Anxiety Pad Revolution is appreciated, as well as the connections of the cables with the aluminium foil inside the module, the pins were included to the Arduino Leonardo, coming from the connections with the cables. 


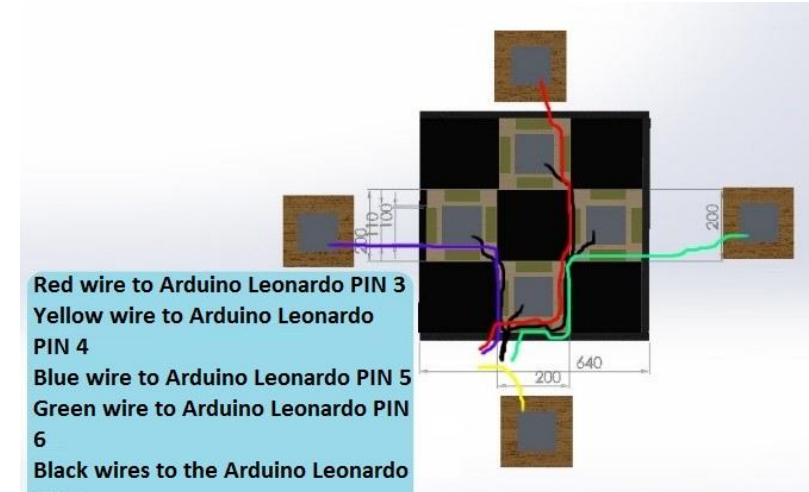

GND

Fig. 6: Assembly Diagram Dance Anti-Anxiety Pad Revolution.

Source: self-made.

\subsection{Biofeedback through an artificial neuronal network implementation in c ++ of a neural network type multi- layer perceptron above Arduino}

An Artificial Neural Network was implemented, using the Multilayer Perceptron algorithm (MLP), in an Arduino microcontroller that allows the latter to learn and predict the behaviour of a user that activates some buttons. This serves as proof of concept of the implementation of artificial neural networks on embedded systems in order to develop a better prediction.

\subsection{Prediction game of anxiety levels}

A simple prediction game is presented in which the Arduino must learn the movements of the user that interacts with it. There are [4] buttons and [4] LEDs (each button corresponds to a button and vice versa). Also included are two additional pushbuttons labelled "TRAIN" and "EVALUATE" and two additional leds labelled "TRAINING FINISHED" and "EVALUATING". An MLP-type neuronal network with 8 inputs, a hidden layer of 8 neurons and an output layer of 4 neurons is implemented. 4 Input neurons collect the inputs of the 4 buttons ( $1=$ pressed, $0=$ not pressed $)$ and the 4 remaining input neurons receive the value of the buttons from the previous moment (it is a way of giving memory to the network), the four output neurons are connected to the 4 output LEDs (ignition $=$ output of the neuron greater than 0.5 , off $=$ otherwise).

In evaluation mode the Arduino, each time it detects the pulsation of a button, applies the values corresponding to the 8 input neurons, evaluates the MLP and emits the value of the 4 output neurons to the LEDs. In training mode, the network trains itself observing the user's pulsations. The mechanics of the "game" is the following: As soon as the Arduino is started, it starts in EVALUATION mode and illuminates the "EVALUATING" led. In this mode, each time one of the [4] input buttons is pressed, the neural network is evaluated and the corresponding output is emitted. As initially the synaptic weights are random, the LEDs will act in a random way depending on the input.

When we want to train the neural network, press "TRAIN". This causes the "EVALUATING" led to go off to indicate that we are in learning mode and the network enters learning mode. The user starts to press the buttons in the order he wants, a sequence of four beats is assumed in such a way that every four beats, the network is trained to be able to learn the sequence. Each time we finish entering a sequence (four clicks), the network is trained with the sequence entered and the LED "TRAINING FINISHED" lights up and if we continue repeating the sequence we will consolidate the learning of the neural network.

Once the "TRAINING FINISHED" led has been illuminated at least once, we can return to the evaluation mode by pressing the "EVALUATE" button. Upon entering the evaluation mode again, the "EVALUATING" led will light up. After training the network will have the synaptic weights modified by the learning in such a way that it will try to predict which button the player will activate at each moment.

\section{Data analysis and processing}

The data obtained from the measurement were processed to the 50 students of the sample, from them the biofeedback techniques were applied, in a first stage this sample was applied the anxiety test standardized by Zung, where it can be seen that a total of 13 students, have a low level of anxiety, 30 students have a medium level of anxiety and a total of 7 students have a very high level of anxiety. It can also be observed taking into account the variable sex, that women have high levels of anxiety compared to men.

Table 1: Level of Anxiety Trait According to the Zung Test

\begin{tabular}{lllll}
\hline Gender & \multicolumn{4}{l}{ Anxiety trait level according to Zung's test } \\
& Low & Medium & High & Total \\
Men & 5 & 12 & 3 & 20 \\
Women & 8 & 18 & 4 & 30 \\
Total & 13 & 30 & 7 & 50 \\
\hline
\end{tabular}

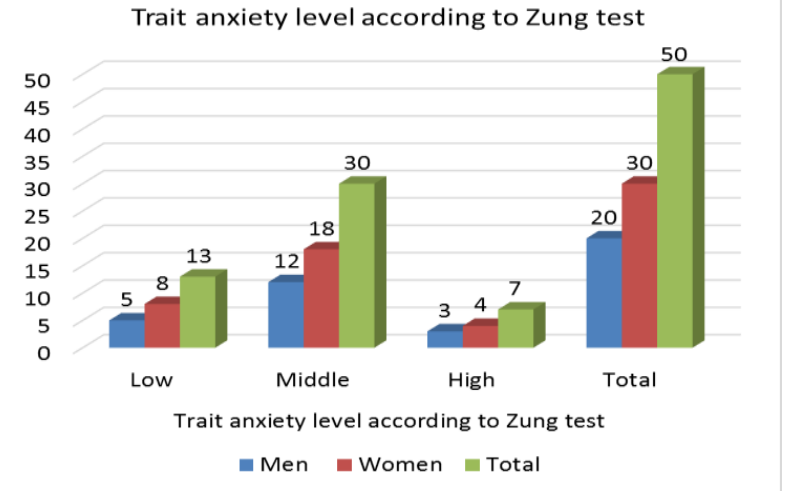

Fig. 7: Levels of Anxiety According to Zung's Test.

Source: self-made

According to the sample of the 50 students who were measured the level of anxiety using our prototype, it can be seen that a total of 17 students have a low level of anxiety, 27 students have an average level of anxiety and a total of 6 students present a high level of anxiety. In Figure 8, as in Figure 7, it can be observed taking into account the sex variable, that women have high anxiety levels compared to men.

Table 2: Level of Anxiety Trait through Our Prototype.

\begin{tabular}{lllll}
\hline Gender & \multicolumn{4}{l}{ Anxiety trait level with prototype } \\
\hline & Low & Medium & High & Total \\
Men & 5 & 13 & 2 & 20 \\
Women & 12 & 14 & 4 & 30 \\
Total & 17 & 27 & 6 & 50 \\
\hline
\end{tabular}

Trait anxiety level according to the prototype proposed

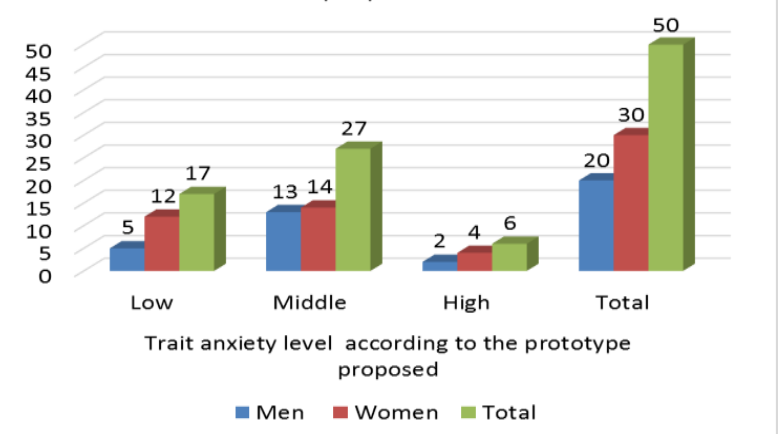

Fig. 8: Levels of Anxiety Applying our Proposal.

Source: self-made 
In the following figure it can be seen that there is little variation between the results obtained through the Zung anxiety test and our proposal.

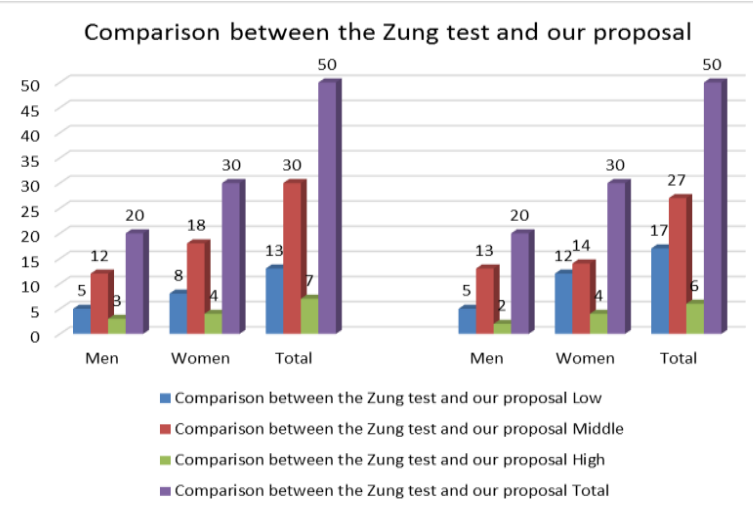

Fig. 9: Comparison of Anxiety Levels between the Zung Test and our Proposal.

Source: self-made

\section{Discussion of results}

The following table shows the results of the comparison between the measurements developed before and after applying the biofeedback techniques implemented, where it can be seen that out of 13 people who previously had a low level of anxiety, now 31 people have a level low anxiety: of the 30 people who previously had an average level of anxiety, now only 14 have an average level of anxiety and out of the 7 people who previously had a high level of anxiety, now only 5 people have this high level of anxiety

Table 3: Comparison of Anxiety Levels after Applying Biofeedback Techniques

\begin{tabular}{llll}
\hline \multirow{2}{*}{ Gender } & $\begin{array}{l}\text { Comparison of anxiety levels after applying Biofeedback tech- } \\
\text { niques }\end{array}$ \\
\hline & Low & & Medium \\
& Before & After & Previous \\
Men & 5 & 12 & 12 \\
Women & 8 & 19 & 18 \\
Total & 13 & 31 & 30 \\
\hline
\end{tabular}

Continuation: table of comparison of anxiety levels after applying Biofeedback techniques.

Table 3: Continued

\begin{tabular}{llll}
\hline Medium & Low & Total \\
\hline After & Before & After & \\
6 & 3 & 2 & 20 \\
8 & 4 & 3 & 30 \\
14 & 7 & 5 & 50 \\
\hline
\end{tabular}

The following figure, through a bar chart, shows the comparison between a before and after measurements developed without the application of biofeedback techniques where a considerable reduction of anxiety levels can be seen.

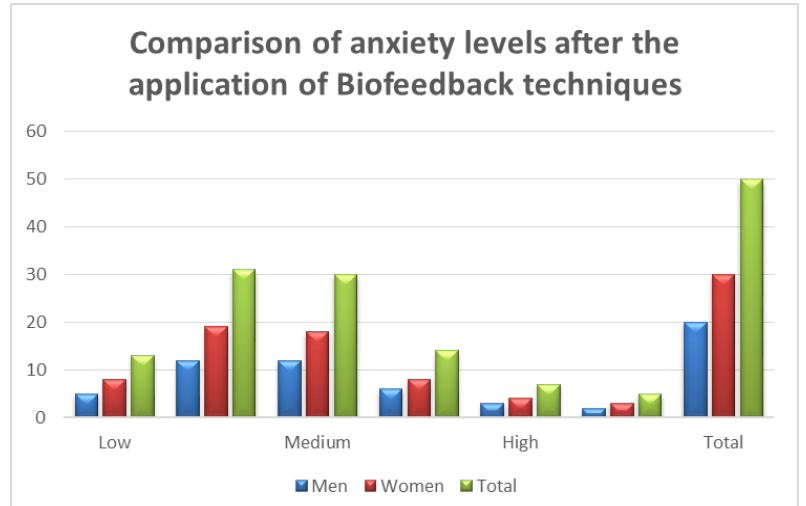

Fig. 10: Comparison of Anxiety Levels after the Application of Biofeedback Techniques

Source: self-made

Table 4: Data Analysis through Descriptive Statistics

\begin{tabular}{|c|c|c|c|c|c|}
\hline Before & & After & & Before & \\
\hline Medium & 8.6 & Medium & 20.6 & Medium & 20 \\
\hline Typical error & 2.3 & Typical error & 5.5 & Typical error & 5.30 \\
\hline Median & 8 & Median & 19 & Median & 18 \\
\hline $\begin{array}{l}\text { Standard devia- } \\
\text { tion }\end{array}$ & 4.0 & $\begin{array}{l}\text { Standard devia- } \\
\text { tion }\end{array}$ & 9.6 & $\begin{array}{l}\text { Standard devia- } \\
\text { tion }\end{array}$ & 9.2 \\
\hline $\begin{array}{l}\text { Sample vari- } \\
\text { ance }\end{array}$ & 16.3 & $\begin{array}{l}\text { Sample vari- } \\
\text { ance }\end{array}$ & 92.3 & $\begin{array}{l}\text { Sample vari- } \\
\text { ance }\end{array}$ & 84 \\
\hline $\begin{array}{l}\text { Asymmetry co- } \\
\text { efficient }\end{array}$ & 0.7 & $\begin{array}{l}\text { Asymmetry co- } \\
\text { efficient }\end{array}$ & 0.76 & $\begin{array}{l}\text { Asymmetry co- } \\
\text { efficient }\end{array}$ & 0.9 \\
\hline Range & 8 & Range & 19 & Range & 18 \\
\hline Minimum & 5 & Minimum & 12 & Minimum & 12 \\
\hline Maximum & 13 & Maximum & 31 & Maximum & 30 \\
\hline Total & 26 & Total & 62 & Total & 60 \\
\hline
\end{tabular}

\begin{tabular}{llllll}
\multicolumn{7}{c}{ Table 4: Continued } \\
\hline After & & Before & & After & \\
\hline Medium & 9.3 & Medium & 4.6 & Medium & 3.3 \\
Typical error & 2.4 & Typical error & 1.2 & Typical error & 0.9 \\
Median & 8 & Median & 4 & Median & 3 \\
$\begin{array}{l}\text { Standard devia- } \\
\text { tion }\end{array}$ & 4.2 & $\begin{array}{l}\text { Standard devia- } \\
\text { tion }\end{array}$ & 2.1 & $\begin{array}{l}\text { Standard devia- } \\
\text { tion }\end{array}$ & 1.5 \\
Sample variance & 17.3 & Sample variance & 4.3 & Sample variance & 2.3 \\
Asymmetry co- & 1.3 & Asymmetry co- & 1.3 & Asymmetry co- & 0.9 \\
efficient & & efficient & & efficient & \\
Range & 8 & Range & 4 & Range & 3 \\
Minimum & 6 & Minimum & 3 & Minimum & 2 \\
Maximum & 14 & Maximum & 7 & Maximum & 5 \\
Total & 28 & Total & 14 & Total & 10 \\
\hline
\end{tabular}

The expected results were satisfactory, since the levels of anxiety and anxiety were considerably reduced, as can be seen in the following table:

The table shows the descriptive statistics of the comparison between a before and after the developed measurements, where it can be seen that there is a decrease between the levels of anxiety between the first and second measurement.

From the Low Anxiety Levels section of the comparison table, it can be seen that before 13 people had low levels of anxiety, later by applying our prototype the results are of 31 people located at a low level of anxiety, so the variation is 18 people with respect to the previous measurement.

From the Average Anxiety Levels section of the comparison chart, it can be seen that before 30 people had average levels of anxiety, later through the application of our prototype the results are of 14 people located at a medium level of anxiety, so the variation is 16 people with respect to the previous measurement.

From the High Anxiety Levels section of the comparison table, it can be seen that before 7 people had high levels of anxiety, later through the application of our prototype the results are of 5 people located at a high level of anxiety, so the variation is 2 people with respect to the previous measurement. 
To know a set of data in detail, it is not enough to know the measures of central tendency, but we also need to know the deviation that the data present in its distribution with respect to the arithmetic average of the mentioned distribution, in order to have a vision of the same ones more in agreement with the reality when describing them reason why the standard deviation is an average of 5.10 .

\section{Conclusions}

It was possible to develop techniques for biofeedback of anxiety states, through automatic detection processes using different available technologies.

The Dance Anti-Anxiety Pad Revolution module is implemented and put into practice in order to develop anti-anxiety dynamics for bodily events.

Scratch software is used to program and design the interface for the Dance Anti-Anxiety Pad Revolution module, including motivating messages, relaxing music and ratings.

A Mobile Application was designed and implemented using the MIT App Inventor platform, which offers an interactive interface, so that students through different tabs can monitor and self-regulate their high levels of anxiety.

The levels of anxiety are considerably reduced, through the application of the different biofeedback techniques developed.

The results of the methodology implemented through experimentation and study of the theoretical framework are validated through the review of the different bibliographical research.

Taking into account the sex variable, women have high levels of anxiety compared to men.

\section{Suggestions to improve the proposal}

Implement an Artificial Neural Network as a complement to the biofeedback techniques proposed. In such a way that chromo therapy can be used, a technique that measures the impact of colours on the organism.

Use and implement sensors that obtain more accurate data to improve the accuracy and effectiveness of monitoring anxiety levels. We would appreciate to send us your suggestions and comments to improve the proposal on the website http://benjaminmaraza.myplace.us/biorretroalimentacion/

\section{Acknowledgement}

A special thanks to the High Performance College of Arequipa for allowing us to experiment with the students and also a thanks to CONCYTEC for having allowed us to carry out the research project which was exposed by my beloved students Alfred and Nicolas in the INTEL-ISEF 2018 out in Pittsburgh-USA.

\section{References}

[1] Arduino como servidor Web_Tutoriales Arduino.html.

[2] Barrajo, M. (2015), IPHealth: Plataforma inteligente basada en open, linked y big data para la toma de decisiones y aprendizaje en el ámbito de la salud, Procesamiento del lenguaje natural.

[3] Basogain. (2012). Redes neuronales artificiales y sus aplicaciones. Madrid: Departamento de Sistema y Automática Escuela Superior de Ingeniería de Bilbao.

[4] Hernández. (2008). Propiedades psicométricas del inventario Zung del estado de ansiedad con mexicanos. Psicología. Avances de la disciplina, 19-46.

[5] Ledesma, S. (2010). Las Redes Neuronales. Fifth Mexican International Conference on Arti. México.

[6] López, J. (2010). Aprendizaje automático. España: RA-MA

[7] Minsky, M. (1989). Redes Neuronales Artificiales. Innovaciones Computacionales, 15.

[8] Prokasy, W., \& Raskin, D. (1973). Electro dermal activity in psychological research. New York: Academic Press.
[9] Rosenblatt, J. (1989). Redes Neuronales, Madrid: española.

[10] Schwartz, M. (1995). New York: Guildford Press. Guildfor Press. pp. 515-544.

[11] Thomson, S. (2017). Los usos de la Biorretroalimentación en psicoterapia. Revista Latinoamericana de Psicología, pp. 60-78. 